\title{
La généralisation algébrique: un processus mathématique peu développé chez les élèves à la fin de l'école secondaire
}

\section{Algebraic generalization: an underdeveloped mathematical process in students at the end of secondary school}

\author{
Hassane Squalli ${ }^{1,2 *}$ \\ ${ }^{1}$ Université de Sherbrooke, Sherbrooke, Québec, Canada, \\ ${ }^{2} \mathrm{OIPA}$ : Observatoire internationale de la pensée algébrique (https://www.oipa.education).
}

\begin{abstract}
Résumé. Généraliser est un processus essentiel de l'activité mathématique. Son apprentissage au primaire et au secondaire ne va pas de soi. Souvent, les élèves construisent en actes des généralités à l'insu de l'enseignant. Ces généralités peuvent être vraies ou fausses sans qu'elles soient questionnées par l'élève et par l'enseignant. Nous faisons l'hypothèse que ce processus est faiblement développé chez les élèves de l'école primaire et secondaire. Pour la vérifier, nous avons mené une recherche auprès d'un échantillon composé de 76 étudiants inscrits dans un programme universitaire de premier cycle et ayant suivi majoritairement une formation au secondaire non spécialisée en mathématiques. Nous leur avons soumis le problème suivant : Si $n$ est un entier naturel, le nombre $n^{2}+n+41$ est-il, 1) toujours premier? 2) quelquefois premier? ou jamais premier? Justifiez votre réponse. L'analyse des réponses porte sur la nature arithmétique ou algébrique de la généralisation ainsi que sur la qualité des justifications. Nos résultats montrent que $75 \%$ des répondants manifestent une généralisation à tendance arithmétique : la généralisation est formulée à partir de quelques essais numériques. Alors que $25 \%$ des réponses manifeste des généralisations à tendance algébrique : la généralisation est formulée à partir d'une analyse de la structure syntaxique de l'expression $n^{2}+n+41$. Ces résultats pointent selon nous une lacune importante de l'enseignement des mathématiques à l'école primaire et secondaire.
\end{abstract}

\begin{abstract}
Generalizing is an essential mathematical process but doesn't have this importance in primary and secondary school. Often, the students construct generalities in action without the knowledge of the teacher. These generalities can be true or false without being questioned by the student and
\end{abstract}

*Corresponding author: Hassane.Squalli@,USherbrooke.ca 
the teacher. We hypothesize that this process is poorly developed in primary and secondary school students. To verify this, we conducted a research on a sample of 76 students enrolled in an undergraduate university program, most of whom had received non-specialized high school training in mathematics. We presented them the following problem: $n$ is a natural number, is the number $n^{2}+n+41,1$ ) always a prime number? 2) sometimes a prime number? or never a prime number? Justify your answer. The analysis of the answers focuses on the arithmetic or algebraic nature of the generalization as well as the quality of the justifications. Our results show that $75 \%$ of the respondents are arithmetic generalization: the generalization is formulated from a few numerical tests. While $25 \%$ of the answers show algebraic generalizations: the generalization is formulated from an analysis of the syntactic structure of the expression $n^{2}+n+41$. These results point out, in our opinion, an important gap in the teaching of mathematics in elementary and secondary school.

\section{Introduction}

Comme l'abstraction, la généralisation est un processus essentiel dans l'activité mathématique. En effet, la plupart des concepts mathématiques sont généraux.

La généralisation est essentielle à la construction des connaissances mathématiques, elle joue donc un rôle fondamental dans l'apprentissage des mathématiques. Sierpinska (1995), [12] y voit un passage obligé au développement de la compréhension mathématique. La généralisation constitue de ce fait un enjeu crucial en enseignement.

Selon Mason (1994) [9] " généraliser c'est tirer des conclusions valables pour tous les cas à partir de quelques exemples.» (p. 7) La généralisation commence dès qu'on pressent un cheminement sous-jacent, même si on est encore incapable de le formuler. Grossièrement, on peut distinguer trois moments importants dans le processus de généralisation: 1) pressentir la généralisation ; 2) formuler la généralisation et 3) justifier la généralisation. La généralisation est à la fois un processus et un produit (de ce processus). Pour une question de clarté, nous parlerons de généralisation pour désigner le processus et de généralité pour désigner le produit (Squalli, 2017) [16]. À l'aide d'une analyse phénoménologique, nous présentons dans ce dernier texte différentes techniques de généralisation.

L'apprentissage de la généralisation au primaire et au secondaire ne va pas de soi. Souvent les élèves construisent en actes des généralités à l'insu de l'enseignant. Ces généralités peuvent être vraies ou fausses sans qu'elles soient questionnées par l'élève et par l'enseignant. Ainsi, un élève peut formuler des généralités à partir de l'examen de quelques cas particuliers, d'un cas pris au hasard ou de l'extension du domaine de validité d'une généralité déjà construite. Par exemple, un élève pourrait constater que $6|246,6| 126 ; 6 \mid 186$ et en conclure que 6 divise tout nombre de 3 chiffres de la forme ab6 (Guzmán; Kieran et Squalli, 2003) [5]. Ou encore, l'élève apprend de sa riche expérience avec la multiplication des nombres entiers naturels, ou de son enseignant, que: « Multiplier un nombre par 10, revient à ajouter 0 à droite de son chiffre des unités ». Il étendra naturellement cette généralité aux nombres décimaux et écrira : $12,3 \times 10=12,30$.

Certains élèves éprouvent beaucoup de difficultés à généraliser (Mary, Squalli et Schmidt, 2008, [8]; Vlassis, Demonty et Squalli, 2017) [20]. Par exemple, nombreux d'entre eux ne voient pas la généralisation implicite dans une situation: ils n'arrivent pas à changer de point de vue, à se détacher de la singularité d'une situation et à voir le général dans le particulier. Et lorsqu'ils arrivent à pressentir et formuler une généralisation, ils éprouvent de la difficulté à la justifier. Par exemple, pour certains élèves un exemple numérique est plus convaincant qu'une démonstration algébrique. D'autres ne se rendent pas compte qu'un seul 
contre-exemple suffit pour réfuter une hypothèse de généralisation. Ces constats pointent selon nous une lacune importante de l'enseignement des mathématiques à l'école primaire et secondaire. Cela nous a conduits à formuler l'hypothèse que la généralisation est un processus faiblement développé chez les élèves de l'école primaire et secondaire. Pour la vérifier, nous avons décidé de questionner un échantillon de personnes composé d'étudiants inscrits dans un programme universitaire de premier cycle et ayant suivi majoritairement une formation au secondaire non spécialisée en mathématiques.

\section{Considérations d'ordre conceptuel}

La généralisation algébrique est une composante essentielle de la pensée algébrique que nous définissons comme une manière de penser dans ce type d'activité faisant intervenir des opérations (lois de composition internes, externes, binaires ou n-aires) pouvant être de nature quelconque (addition, multiplication, translation, etc.), mais répétées un nombre fini de fois. (Squalli, 2000,) [13]. Sur le plan opératoire, la pensée algébrique se déploie au moyen de 1) un ensemble de raisonnements particuliers (comme généraliser, raisonner de manière analytique, symboliser et opérer sur des symboles; exprimer, interpréter, raisonner sur des relations entre variables, en particulier des relations fonctionnelles; raisonner en termes de structures; 2) manières d'approcher des concepts en jeu dans les activités algébriques; voir l'égalité comme une relation d'équivalence; laisser les opérations en suspens; voir une expression numérique comme un objet en soi et non uniquement comme une chaîne de calcul; etc. et 3) des modes de représentation et des manières d'opérer sur ces représentations.

Plusieurs auteurs distinguent deux formes de généralisation: 1) la généralisation empirique (Dörfler, 1991,) [3] ou inductive (Piaget et Henriques, 1978,) [10] et 2) la généralisation théorique (Dörfler, 1991) [3] ou constructive (Piaget et Henriques, 1978, [10]). Comme l'expliquent Piaget et Henriques (1978) [10]:

[La généralisation inductive] part des observables attachés aux objets, donc d'abstractions empiriques, et s'en tient à eux pour vérifier la validité des relations observées, pour établir leur degré de généralité et en tirer des prévisions ultérieures (mais sans encore chercher d'explication ou de "raison" ce qui conduirait à dépasser les observables), est alors de nature essentiellement extensionnelle et consiste à procéder du « quelque» au «tous» ou du «jusqu'ici» au «toujours» (...).

[La généralisation constructive] s'appuie ou porte sur les opérations du sujet ou leurs produits, elle est en ce cas de nature simultanément compréhensive et extensionnelle (...). (p.6)

Pour illustrer ces deux formes de généralisation, considérons le problème suivant, discuté en détail dans (Squalli, 2017,) [16] : Quels sont les entiers qui possèdent un nombre impair de diviseurs?

Une démarche possible d'une généralisation empirique, ou inductive, peut être décrite ainsi. En déterminant le nombre de diviseurs d'une liste de nombres, on peut voir surgir une régularité en cherchant à découvrir ce qu'ont en commun les nombres ayant un nombre impair de diviseurs. Ainsi, après avoir généré le tableau suivant :

Tableau 1 : génération des nombres de diviseurs.

\begin{tabular}{|l|l|l|l|l|l|l|l|l|l|l|l|l|}
\hline Nombres & 1 & 2 & 3 & 4 & 5 & 6 & $\ldots$ & 9 & $\ldots$ & 16 & $\ldots$ & 25 \\
\hline $\begin{array}{l}\text { Nombre de } \\
\text { diviseurs }\end{array}$ & 1 & 2 & 2 & 3 & 2 & 4 & $\ldots$ & 3 & $\ldots$ & 5 & $\ldots$ & 3 \\
\hline
\end{tabular}


Il apparaît que les nombres entre 1 et 25 qui ont un nombre impair de diviseurs sont 1, 4, 9, 16 et 25 . On peut alors se demander ce que ces nombres ont en commun. Un raisonnement inductif nous conduit à conjecturer que les nombres ayant un nombre impair de diviseurs sont les carrés parfaits. La généralité est inférée à partir d'un nombre restreint d'exemples, et se base sur les qualités nombrantes des exemples examinés. Elle est dans ce sens empirique (ou inductive). En outre, notre stratégie ne nous permet pas de comprendre pourquoi les nombres carrés, et uniquement cette catégorie de nombres, possèdent un nombre impair de diviseurs. Cette stratégie de généralisation est la plus naturelle chez les élèves. Le nombre d'exemples générés avant de voir une régularité dépend de l'expérience des élèves dans ce type d'activités. Certains auront besoin d'un grand nombre d'exemples avant de pressentir une régularité.

Une démarche possible de généralisation théorique, ou constructive, peut être décrite ainsi : En examinant la structure des diviseurs d'un nombre, disons $n$, on remarque que si $d$ est un diviseur de $n$, alors $d^{\prime}=\frac{n}{d}$ est aussi un diviseur de $n$ et que $d$ et d'sont symétriquement placés par rapport à $\sqrt{n}$. Quand le nombre de diviseurs de $n$ est impair, alors il existe forcément un diviseur $d$ double $: d \times d=n$ et donc $\mathrm{d}=\sqrt{n} . \sqrt{n}$ est donc un entier, autrement dit, $n$ est un carré parfait. Inversement, si $n$ est un carré parfait, $\sqrt{n}$ est un diviseur (double) de $n$, le nombre des diviseurs de $\mathrm{n}$ est donc forcément impair. Cette généralisation est théorique, ou constructive, car elle se base sur les opérations du sujet et non sur les observables empiriques. Elle permet en outre de nous assurer de la validité de la généralisation par une argumentation intellectuelle et de comprendre la raison qui explique cette généralité : les diviseurs d'un nombre $n$ sont symétriquement placés par rapport à $\sqrt{n}$ (une symétrie de nature multiplicative) le nombre des diviseurs de $n$ est impaire SSI $\sqrt{n}$ est un entier, autrement dit n est un carré parfait.

Cette distinction entre ces deux formes de généralisation nous permet de distinguer entre une généralisation arithmétique et une généralisation algébrique. En effet, dans notre conceptualisation (Squalli, 2015, 2020, [15, 17]), si l'arithmétique peut être définie comme la science des nombres, des quantités et des grandeurs (Carraher et Schliemann, 2007, [2]), l'algèbre peut être définie comme la science des opérations. Le caractère algébrique de la pensée ou de l'activité mathématique ne réside pas dans la présence de signes alphanumériques, mais dans les significations des concepts ainsi que dans la nature des raisonnements impliqués. Dans ce sens, une généralisation est arithmétique si elle est empirique, autrement dit si la généralité est induite à partir de quelques exemples numériques et fondée sur la qualité nombrante de ces exemples. Une généralisation est algébrique si elle est théorique, autrement dit si elle s'appuie sur un raisonnement du sujet basé sur une argumentation intellectuelle.

\section{Analyse a priori du problème à l'étude}

Le problème objet de cette étude est le suivant :

Si n est un entier naturel, le nombre $n^{2}+n+41$ est-il,

1) toujours premier? 2) quelquefois premier? ou 3) jamais premier? Justifiez votre réponse.

Le nombre $n^{2}+n+41$ est intéressant car il est premier pour $n=0,1,2,3, \ldots, 38,39$. On peut donc s'attendre à ce que les étudiants qui ont une tendance à généraliser de manière arithmétique de répondre que ce nombre est toujours premier. En revanche, en observant la structure syntaxique de l'expression $n^{2}+n+41$ on peut comprendre que cette expression numérique génère un nombre multiple de 41 pour $n=41$. Écrite sous cette forme équivalente, $n(n+1)+41$ génère également un multiple de 41 pour $n=40$. On peut donc s'attendre 
que à ce que les étudiants qui ont une tendance à généraliser de manière algébrique de répondre que ce nombre est quelquefois premier et que leur argument soit fondé sur une analyse syntaxique de l'expression $n^{2}+n+41$.

\section{Considérations d'ordre méthodologiques}

Le problème précédent a été administré à un échantillon composé de 76 étudiants en $3^{\mathrm{e}}$ année du programme baccalauréat en adaptation scolaire et sociale lors de la première séance d'un cours en didactique de l'algèbre. D'une durée de 4 ans, ce programme universitaire forme des enseignants spécialisés qui interviennent auprès des élèves à risque, handicapés ou en difficulté d'adaptation ou d'apprentissage en vue de favoriser leur réussite personnelle, scolaire et sociale. Les étudiants admis dans ce programme détiennent un diplôme d'études collégiales $(\mathrm{DEC})^{\dagger}$. Ils ont donc réussi 13 années de scolarité avant leur entrée à l'université. Leur formation mathématique a été forgée par les cours de mathématiques à l'école primaire, secondaire et collégiale. À partir du secondaire 4 et au collège ils ont généralement suivi les cours de mathématiques obligatoires pour l'obtention du DEC. La majorité d'entre eux ont suivi une filière en sciences humaines. La formation mathématique de ces étudiants nous semble ainsi assez représentative de la formation mathématique de base de la population large de personnes qui ont complété leurs études primaires, secondaires et collégiales. Cet échantillon est donc pertinent pour vérifier notre hypothèse : au terme de la scolarité à l'école secondaire, le processus de généralisation algébrique est faiblement développé chez les élèves.

Les étudiants devaient répondre au problème de manière individuelle. Ils avaient en leur possession la liste des nombres premiers de 2 à 1999, et une calculatrice pour faire des calculs, éventuellement.

\section{Résultats}

L'analyse des réponses des étudiants porte dans un premier temps sur la nature arithmétique ou algébrique de la démarche de généralisation. Rappelons qu'une généralisation est dite à arithmétique (Ar) si la généralité est formulée à partir de quelques essais numériques. Elle sera dite à algébrique ( $\mathrm{Al})$ si la généralité est formulée à partir d'une analyse de la structure syntaxique de l'expression $n^{2}+n+41$.

Le tableau 2 suivant présente la répartition des réponses selon les catégories Ar et Al.

Tableau .2: Nature des généralisations.

\begin{tabular}{|l|l|}
\hline Ar & Al \\
\hline $75 \%$ & $25 \%$ \\
$(57)$ & $(19)$ \\
\hline
\end{tabular}

Il en ressort que trois quarts du nombre des étudiants a utilisé une généralisation arithmétique. La tendance à calculer, à inférer la régularité à partir de quelques essais numériques est donc plus forte chez ce groupe d'étudiants que de s'abstenir de calculer et d'analyser la structure syntaxique de l'expression. Cette conduite, propre d'une culture arithmétique, ne serait surprenante chez des élèves du primaire ou du début du secondaire. Car comme le soulignent plusieurs auteurs (Booth, 1984) [1], (Vergnaud, 1986) [19], (Filloy et Rojano, 1989) [4] et (Kieran, 1994) [7], les longs apprentissages qu'ont réalisés les élèves en arithmétique

\footnotetext{
$\dagger$ Dans le système scolaire québécois, l'ordre primaire est d'une durée de 6 ans, le secondaire de 5 ans. Le collégial est un ordre préuniversitaire d'une durée de 2 ans pour la formation générale et de 3 ans pour la formation professionnelle.
} 
viennent faire obstacle au passage d'un mode de pensée arithmétique à un mode de pensée algébrique.

Le tableau 3suivant présente la répartition des réponses dans chacune des deux catégories.

Tableau 3 : Répartition des réponses dans les deux catégories Ar et Al.

\begin{tabular}{|c|c|c|c|c|}
\hline & $\begin{array}{c}\text { Quelque fois } \\
\text { premier }\end{array}$ & Toujours premier & Jamais premier & Total \\
\hline $\mathrm{Ar}$ & $43,9 \%$ & $54,4 \%$ & $1,7 \%$ & $100 \%$ \\
& $(25)$ & $(31)$ & $(1)$ & $(57)$ \\
\hline $\mathrm{Al}$ & $21,1 \%$ & $73,7 \%$ & $5,2 \%$ & $100 \%$ \\
& $(4)$ & $(14)$ & $(1)$ & $(19)$ \\
\hline
\end{tabular}

En cohérence avec notre analyse a priori, on aurait pu s'attendre à ce que la majorité des étudiants de la catégorie Ar répondent que le nombre $n^{2}+n+41$ est toujours premier et que la majorité des étudiants de la catégorie $\mathrm{Al}$ répondent que le nombre $n^{2}+n+41$ est quelque fois premier. Or, le tableau 2 montre que dans la catégorie Ar, presque $44 \%$ des étudiants ont répondu Quelque fois premier et un peu plus de 54\% ont répondu toujours premier. Cela signifie que dans leurs essais numériques, les premiers ont testé une valeur de $\mathrm{n}$ pour laquelle le nombre le nombre $n^{2}+n+41$ est composé; alors que chez les seconds, tous les essais ont abouti à des nombres premiers. Mais ce qui est encore plus surprenant dans ces résultats est que presque les trois quarts des étudiants de la catégorie $\mathrm{Al}$ ont répondu toujours premier. Analysons plus finement les réponses des étudiants pour mieux comprendre ces résultats.

\section{Analyse des réponses de la catégorie $\operatorname{Ar}(\mathbf{N}=57)$}

Nous cherchons ici à comprendre la logique derrière les choix des nombres utilisés comme valeur de $n$ dans l'expression $n^{2}+n+41$. Deux étudiants ont fait des erreurs de calcul. Huit étudiants ont attribué des valeurs différentes à $n$ dans les monômes $n$ et $n^{2}$ ! Cela montre le niveau faible des compétences algébriques de ces étudiants.

Chez les autres étudiants de cette catégorie, nous avons identifié 4 types de conduites.

Le premier consiste à choisir la valeur des nombres testés selon un ordre croissant en commençant par 1 ou 2. Par exemple, utilisant les nombres 2, 3 et 4 comme valeur de n, l'étudiante (S39) obtient à chaque fois un nombre premier, elle généralise cette régularité à tous les nombres en concluant « alors $n^{2}+n+41$ est toujours premier ». Un autre étudiant (S9) a un raisonnement semblable. Après avoir calculé la valeur de l'expression $n^{2}+n+41$ pour $n=1,2,3,4$ il conclue que « suivant cette logique, cette formule donnera toujours des nombres premiers ». Dans cette forme de généralisation par répétition (Squalli, 2017, [16]) la répétition de l'observable (le nombre obtenu est premier) pour des valeurs différentes de la variable $n$ pousse à postuler qu'il en sera toujours ainsi. La généralité est obtenue par extension du domaine de validité de l'observable. La vraisemblance de la conclusion tient à la constance de l'observable alors que les valeurs de la variable varient.

Inférer la régularité d'une suite numérique à partir de l'examen de quelques-uns de ses termes successifs est une stratégie de généralisation répandue chez les élèves du secondaire et encouragée en enseignement. La tâche suivante est un spécimen de tâches que l'on peut trouver dans un manuel scolaire au début du secondaire :

\section{5, 9, 13, 17, 21 sont les cinq premiers termes d'une suite numérique.}




\section{Trouver le terme suivant, ensuite le terme de rang $n$.}

La réponse attendue et que le sixième terme est 25 et le terme de rang $n$ est $1+4 n$.

Or cette généralisation est erronée, puisqu'il existe une infinité de suites numériques dont les cinq premiers termes sont, 5, 9, 13,17, 21. Par exemple les deux suites suivantes :

$$
\begin{gathered}
u_{n}=1+4 n \text { pour } n=1,2,3,4,5 \text { et } u_{n}=0 \text { pour } n \geq 6 \\
v_{n}=1+4 n+(n-1)(n-2)(n-3)(n-4)(n-5), n \geq 1
\end{gathered}
$$

Un second type de conduite des étudiants est de choisir les valeurs de n selon les propriétés particulières de certaines catégories de nombres; par exemple en variant les choix de nombres selon leur parité (pair/impair), leur taille (petit/grand) ou selon leur primalité (premier/composé). La généralité est attestée dans un premier temps dans chacune des deux catégories de nombres (pair/impair ; petit/grand, premier/composé) et ensuite attestée pour tous les nombres du fait que ces deux catégories forment deux classes disjointes, partitionnant l'ensemble des nombres. La vraisemblance de la généralité tient à la constance de l'observable dans deux catégories complémentaires de nombres. Mais dans chacune de ces catégories, la généralité est obtenue par l'examen de quelques cas particuliers. Le choix des nombres peut suivre la logique du premier type ou encore choisie au hasard, comme le troisième type des conduites des élèves. Le fait de considérer des cas des deux classes de nombres complémentaires semble renforcer la conviction de l'étudiant dans la vraisemblance de la généralité.

Un troisième groupe d'étudiants semble avoir choisi au hasard les valeurs de $n$ dans leurs essais. Or ce choix nous semble volontaire; sa logique serait fondée sur l'idée suivante : un nombre choisi au hasard est aussi peu particulier que possible, il constitue un bon représentant d'un nombre quelconque. Une variable est ainsi perçue ainsi comme représentée par une de ses instanciations choisies au hasard. Nous avons observé cette conduite chez certains de nos étudiants lors de la résolution de tâches de généralisation. Lorsque nous leur demandons comment ils pouvaient être certains que leur généralité formulée à partir de quelques essais numériques est vraie pour tous les nombres, ils nous donnent une réponse du type : «Donnezmoi un nombre quelconque, et je vous montre que c'est vrai ».

Le quatrième est dernier groupe de cette catégorie est composé des étudiants $(\mathrm{N}=25)$ qui ont donné la bonne réponse : le nombre $n^{2}+n+41$ est quelque fois premier. Ces étudiants ont choisi le nombre 41 parmi leurs essais, 2 de ces étudiants ont choisi les nombres 40 et 41 . La question importante ici est de comprendre la logique derrière le choix des valeurs 41 et 40 , lesquels aboutissent au constat que le nombre $n^{2}+n+41$ est composé. Ces choix pourraient être inscrits dans un raisonnement algébrique. En effet, une analyse de la structure syntaxique de l'expression $n^{2}+n+41$ pourrait amener l'étudiant à observer que si $n$ prend la valeur 41 , on obtient un trinôme donc chaque élément est multiple de 41 . La valeur de ce trinôme serait donc un nombre multiple de 41. Le cas du choix de la valeur 40 est plus subtile, puisqu'il nécessite au préalable la factorisation $n^{2}+n=n(n+1)$ qui monte que $n^{2}+n$ est un multiple de 41 quand $n=40$.

Or, nous avons classé les généralisations de ces étudiants dans la catégorie Ar pour les raisons suivantes.

Les étudiants qui ont choisi le nombre 41 ont réalisé le calcul de $41^{2}+41+41=1763$ et vérifié la non primalité de 1763 . Un raisonnement algébrique aurait permis de conclure du caractère composé de $41^{2}+41+41$ sans effectuation du calcul. C'est le cas par exemple de l'étudiant S41. Après avoir réalisé des essais avec les nombres 1, 2, 3, 5 et 10, l'étudiant essaie le nombre $41:$ : $41+1681+41=1763 \mathrm{AH}$ ! AH ! ». Ce contre-exemple lui permet de conclure que le nombre $n^{2}+n+41$ est quelquefois premier. 
Dans le même sens, les deux étudiants qui ont choisi le nombre 40, non pas utilisé la sans factorisation $40^{2}+40=40(40+1)$, mais réalisé le calcul $40^{2}+40+41=1681$ puis vérifié la non primalité de 1681 .

Une hypothèse explicative du choix des nombres 40 et 41 est le fait que les étudiants de cette catégorie semblent choisir naturellement les valeurs de leurs essais dans l'intervalle [1, 41], $\mathrm{du}$ fait que 41 figure dans l'expression $n^{2}+n+41$, et qu'il soit un nombre relativement grand, il agit comme une borne supérieure des valeurs des nombres testés.

\section{Analyse des réponses de la catégorie $\mathrm{Al}(\mathrm{N}=19)$}

Les étudiants de la catégorie 2 se basent dans leurs réponses sur des raisonnements s'appuyant sur une analyse de la structure syntaxique de l'expression. Nous avons identifié trois types de raisonnement.

Le premier type fonde la généralité sur une autre généralité qui est erronée. Par exemple, trois étudiants ont utilisé ce raisonnement: $« n^{2}+n+41$ est toujours un nombre premier car $n^{2}+n$ est toujours un nombre pair et un nombre pair + un nombre premier est un nombre premier ». Voici d'autres exemples de raisonnement basés sur d'autres généralités erronées : « car un nombre non premier + un nombre premier donne un nombre premier $(\mathrm{N}=3)$ » « car un nombre premier + n'importe quel autre nombre est un nombre premier $(\mathrm{N}=2)$ » « $n^{2}+n+41$ est toujours un nombre premier car c'est la somme d'un nombre pair $\left(n^{2}+n\right)$ est d'un nombre impair $(\mathrm{N}=1)$.

Ces raisonnements illustrent le faible niveau de développement du processus de généralisation chez ces étudiants et dévoilent des généralités en actes erronées construites à l'insu de l'enseignant, non explicitées.

Un deuxième type de raisonnement est basé sur une interprétation du nombre $\mathrm{n}$ comme une inconnue et l'expression $n^{2}+n+41$ comme une équation à résoudre $(\mathrm{N}=2)$.

Voici une copie des traces des réponses de ces deux étudiants.

\begin{tabular}{|c|c|}
\hline $\begin{array}{l}\text { Étudiant S17 } \\
\qquad \begin{array}{l}n^{2}+n+41 \\
n^{2}+n=41 \\
n+n=\frac{\sqrt{-41}}{2}\end{array} \\
\text { ce nombre ne sera jamais premier } \\
\text { puisque n n'a pas la valeur d'un nombre } \\
\text { premier }\end{array}$ & 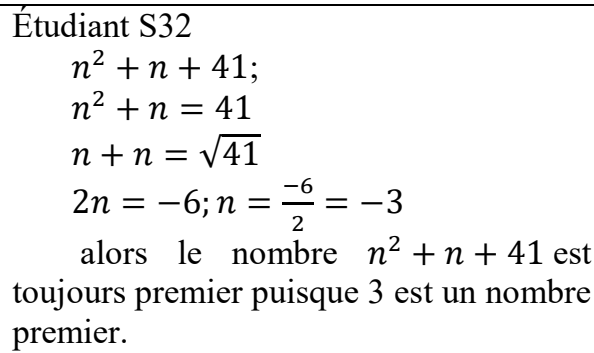 \\
\hline
\end{tabular}

Nous retrouvons cette conduite chez des élèves en difficulté d'apprentissage (Squalli et Anger, 2003) [14] qui éprouvent des difficultés en algèbre élémentaire. Une des hypothèses explicatives de cette conduite proposée par ces deux auteurs est la suivante. Pour ces étudiants, la résolution d'un problème doit aboutir à une valeur numérique simple, la valeur de l'inconnue $n$, en exécutant une série de calculs. Or, l'expression $n^{2}+n+41$ est en quelque sorte inachevée car elle comporte deux opérations «non effectuées». En mettant le signe $=$ à la place du signe + , ces étudiants ôtent cette contrainte puisqu'un calcul devient maintenant possible, et la situation devient compatible avec l'interprétation de $\mathrm{n}$ comme une inconnue dont on cherche à déterminer la valeur.

Une fois l'équation $n^{2}+n=41$ obtenue, ces étudiants tentent d'isoler $\mathrm{n}$ pour trouver sa valeur, en utilisant des transformations erronées montrant l'ampleur de leurs difficultés en algèbre! 
Un seul étudiant a produit la trace d'un raisonnement «partiellement» correct : «Le nombre total de $n^{2}+n+41$ n'est pas premier seulement lorsque ce nombre est divisible par 41 . Toutes les autres fois, il est premier». L'absence de calcul, renforce notre interprétation : l'étudiant est arrivé à la conclusion que le nombre $n^{2}+n+41$ est divisible par 41 (quand $n$ = 41) par une analyse de la structure syntaxique de cette expression. Mais la dernière partie est toutefois une généralisation erronée.

\section{Conclusion}

Dans cette étude nous voulions vérifier l'hypothèse selon laquelle les écoles primaire et secondaire ne préparent pas suffisamment les élèves à développer le processus de généralisation algébrique. Bien que cette étude soit limitée par la nature et la taille de l'échantillon des personnes questionnées, par la nature de la tâche qui n'est pas représentative de l'éventail des tâches de généralisations, elle aura toutefois permis de révéler plusieurs conduites étonnantes des étudiants illustrant leur faible développement du processus de généralisation algébrique. Nous avons vu que les trois quarts de notre échantillon utilisent une généralisation arithmétique, induisant la généralité à partir de quelques essais numériques. La majorité de ceux qui recourent à une généralisation algébrique se base sur des généralités erronées ou dénature complètement le sens de la tâche en transformant l'expression $n^{2}+n+41$ en une équation à résoudre $n^{2}+n=41$. Les analyses ont aussi permis de mettre en évidence certaines significations particulières que donnent certains étudiants à la notion de variable. Pour certains, un nombre choisi au hasard représente n'importe quel nombre (du domaine en jeu); pour d'autres, il suffit de raisonner sur des représentants de deux catégories de nombres, disjointes et partitionnant le domaine des nombres en jeu pour couvrir toutes les valeurs possibles de la variable. Le recours à des exemples numériques reste pour ces étudiants la stratégie privilégiée pour identifier une régularité et la justifier. Ces faiblesses pointent, selon nous, un faible développement de la pensée algébrique chez ces étudiants, dont la généralisation algébrique est une composante essentielle. En effet, dans la réalisation de cette tâche de généralisation, nous avons noté chez les étudiants de notre échantillon des lacunes sur les plans des raisonnements, des significations des concepts et des modes de représentation et des manières d'opérer sur ces représentations. La formation mathématique des étudiants de notre échantillon à l'école primaire et à l'école secondaire s'inscrit dans une approche transitionnelle de l'arithmétique à l'algèbre : avant d'aborder l'algèbre, il faut une bonne base en arithmétique ; aussi, l'algèbre ne pourrait être véritablement abordée qu'à l'école secondaire, une fois que les élèves ont acquis suffisamment d'expérience en arithmétique, une base sur laquelle va s'ériger la construction du savoir algébrique. Mais le passage pour les élèves d'un mode de pensée arithmétique à un mode de pensée algébrique est loin d'être facile à réaliser et pose problème (Vergnaud, Cortes et Favres-Artigue, 1988) [18], (Kieran, 1992) [6], (Rojano, 1996) [11]. Dans leur article synthèse, (Carraher et Schliemann, 2007) [2] présentent des exemples de telles difficultés documentées par la recherche.

Cette étude montre ainsi toute la pertinence de la perspective early algebra, laquelle réfère à la fois à un domaine de recherche, une perspective curriculaire et un domaine de formation des enseignants (Carraher et Schliemann, 2007) [2]. Cette perspective est basée sur l'idée de favoriser le développement précoce de la pensée algébrique, c'est-à-dire depuis les premières années du primaire. Cette tendance curriculaire est actuellement suivie par un nombre de plus en plus grand de pays et régions du monde (Squalli, 2020) [17]. 


\section{Références}

1. Booth, L. (1984). Algebra: children's strategies and errors. Windsor: NFER-NELSON.

2. Carraher, D.W. et Schliemann, A.D. (2007). Early algebra and algebraic reasoning. In F. K. Lester (dir.), Second handbook of research on mathematics teaching and learning: A project of the National Council of Teachers of Mathematics. Vol II. (p. 707-762). Greenwich, CT : Information Age Publishing.

3. Dörfler, W. (1991). Forms and means of generalization in mathematics, dans A. J. Bishop, S. Mellin-Olsen and J. Van Dormolen (Eds.), Mathematical knowledge: its growth through teaching, Dordrecht, Kluwer Academic Publishers.p. 63-85.

4. Filloy, E. \& Rojano, T. (1989). Solving equation: The transition form arithmetic to algebra. For the Learning of Mathematics, volume 9, n. 2, pp. 19-25

5. Guzmán, J. ; Kieran C. et Squalli, H. (2003). La calculadora con pantalla multilínea y el surgimiento de estrategias numéricas en alumnos de primero, segundo y tercer año de secundaria. Educacion matematica, 15(2), p. 105-127.

6. Kieran, C. (1992). The learning and teaching of school algebra. In A. D. Grouws (Ed.), Handbook of Research on Mathematics Teaching and Learning (pp. 390-419). Reston, VA: National Council of Teachers of MathematicsLins, 1992.

7. Kieran, C. (1994). A functional approach to the introduction of algebra - Some pro and cons. In J. Ponte \& J. F. Matos (Eds.), Proceedings of The Eighteen International Conference for the Psychology of Mathematical Education, volume 1, pp. 157-175. Lisbon: University of Lisbon.

8. Mary, C., Squalli, H. et Schmidt, S. (2008). Mathématiques et élèves en difficulté grave d'apprentissage : contexte favorable à l'interaction et au raisonnement mathématique. In. J.-M. Bisaillon et N. Rousseau. Les contextes d'intervention favorables aux jeunes en grandes difficultés (p. 167-192), Montréal : Presses de l’Université du Québec.

9. Mason, J. (1994) L’esprit mathématique. Montréal : Modulo éditeur

10. Piaget, P. et G. Henriques. (1978). Recherches sur la généralisation. Paris : Presses Universitaires de France

11. Rojano, T. (1996). Developing algebraic aspects of problem solving within a spreadsheet environment. In Approaches to algebra (pp. 137-145). Springer, Dordrecht.

12. Sierpinska, A. (1995) La compréhension en mathématiques. Montréal, Modulo Éditeur : Collection La Spirale.

13. Squalli, H. (2000). Une reconceptualisation du curriculum d'algèbre dans l'éducation de base. Thèse de doctorat. Québec, Université Laval.

14. Squalli, H. et Angers, R. (2003). Une évaluation diagnostique en algèbre élémentaire : le cas de Jeanne. Revue de l'ADOQ. 15(2). 4-8

15. Squalli, H. (2015). La généralisation algébrique comme abstraction d'invariants essentiels. In Theis L. (Ed.) Pluralités culturelles et universalité des mathématiques : enjeux et perspectives pour leur enseignement et leur apprentissage Actes du colloque Espace Mathématique Francophone 2015-GT 3

16. Squalli, H. (2017), La généralisation algébrique : une analyse phénoménologique. Revue Marocaine de Didactique des Mathématiques, v. 2, p. 20-27, june 2017. ISSN 25505424. téleaccssible:

$<$ http://www.geotop.org/journal/index.php/RMDM/article/view/43>.

17. Squalli, H. (2020) Early algebra : genèse d'un domaine de recherche, évolution et perspectives. In Squalli, H., Oliveira, I., Bronner, A. et Larguier, M. (2020). Le 
développement de la pensée algébrique à l'école primaire et au début du secondaire. Recherches et perspectives curriculaires. Québec : Livres en ligne du CRIRES. https ://lel.crires.ulaval.ca/oeuvre/le-developpement-de-la-pensee- algebrique-lecoleprimaire-et-au-debut-du-secondaire-recherches.

18. Vergnaud, G., Cortes, A. \& Favres-Artigue, P. (1988). Introduction de l'algèbre auprès des débutants faibles; problèmes épistémologiques et didactiques. Dans. Gérard Vergnaud, Guy Brousseau et Miche Hulin (Eds.) Didactique et acquisition des connaissances scientifiques. Actes du Coloque de Sèvre, mai 1987, pp. 259-280. Paris : Éditions La pensée Sauvage.

19. Vergnaud, G. (1986), Long terme et court terme dans l'apprentissage de l'algèbre in Actes du premier colloque franco-allemand de didactique, Éditions La Pensée Sauvage.

20. Vlassis, J., Demonty, I. et Squalli, H. (2017). Développer la pensée algébrique à travers une activité de généralisation basée sur des motifs (patterns) figuratifs. Nouveaux cahiers de la recherche en éducation,20(3), 131-155. https://doi.org/10.7202/1055731ar. 